\title{
Smoothed state estimates under abrupt changes using sum-of-norms regularization
}

\author{
Henrik Ohlsson, Fredrik Gustafsson, Lennart Ljung and Stephen Boyd
}

\section{Linköping University Post Print}

N.B.: When citing this work, cite the original article.

Original Publication:

Henrik Ohlsson, Fredrik Gustafsson, Lennart Ljung and Stephen Boyd, Smoothed state estimates under abrupt changes using sum-of-norms regularization, 2012, Automatica, (48), 4, 595-605.

http://dx.doi.org/10.1016/j.automatica.2011.08.063

Copyright: Elsevier

http://www.elsevier.com/

Postprint available at: Linköping University Electronic Press

http://urn.kb.se/resolve?urn=urn:nbn:se:liu:diva-60985 


\title{
Smoothed State Estimates Under Abrupt Changes Using Sum-of-Norms Regularization *
}

\author{
Henrik Ohlsson $^{\text {a }}$, Fredrik Gustafsson ${ }^{\text {a }}$, Lennart Ljung ${ }^{\mathrm{a}}$, Stephen Boyd ${ }^{\mathrm{b}}$

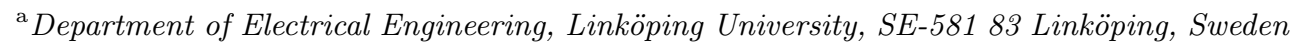 \\ ${ }^{\mathrm{b}}$ Department of Electrical Engineering, Stanford University, Stanford, CA 94305 USA
}

\begin{abstract}
The presence of abrupt changes, such as impulsive and load disturbances, commonly occur in applications, but make the state estimation problem considerably more difficult than in the standard setting with Gaussian process disturbance. Abrupt changes often introduce a jump in the state, and the problem is therefore readily and often treated by change detection techniques. In this paper, we take a different approach. The state smoothing problem for linear state space models is here formulated as a constrained least-squares problem with sum-of-norms regularization, a generalization of $\ell_{1}$-regularization. This novel formulation can be seen as a convex relaxation of the well known generalized likelihood ratio method by Willsky and Jones. Another nice property of the suggested formulation is that it only has one tuning parameter, the regularization constant which is used to trade off fit and the number of jumps. Good practical choices of this parameter along with an extension to nonlinear state space models are given.
\end{abstract}

Key words: state estimation, impulsive disturbance, load disturbance, smoothing, sparsity, regularization, change detection

\section{Introduction}

We consider the problem of state estimation in linear state space models, where impulsive disturbances occur in the process model. The case of impulsive process disturbances occurs frequently in at least three different application areas.

- In automatic control, impulsive disturbance is often used to model load disturbances.

- In target tracking, impulsive disturbances are used to model force disturbances, corresponding to maneuvers for the tracked object.

- In Fault Detection and Isolation (FDI) literature impulsive disturbance is used to model additive faults.

\footnotetext{
* Partially supported by the Swedish foundation for strategic research in the center MOVIII and by the Swedish Research Council in the Linnaeus center CADICS. Also partial support (Ljung and Ohlsson) from the European Research Council under the advanced grant LEARN, contract 267381, which is gratefully acknowledged.

Email addresses: ohlsson@isy.liu.se (Henrik Ohlsson), fredrik@isy.liu.se (Fredrik Gustafsson), ljung@isy.liu.se (Lennart Ljung), boyd@stanford.edu (Stephen Boyd).
}

Usually, this is done in a deterministic setting [30], but a stochastic framework is also common $[2,17]$.

There are several conceptually different ways to handle disturbances in state estimation. We shall review and discuss both deterministic and stochastic models.

The standard linear state space model with disturbances is

$$
\begin{aligned}
x(t+1) & =A x(t)+B u(t)+G v(t) \\
y(t) & =C x(t)+e(t),
\end{aligned}
$$

where $x(t)$ is the state, $u(t)$ is the input, and $y(t)$ is the output at time $t$. Here $e$ is the measurement noise and $v$ is the process disturbance. It is natural in many applications to model the measurement noise as white noise (a sequence of independent random vectors):

$$
\begin{aligned}
E\left[e(t) e^{T}(s)\right] & =0 \quad \text { if } t \neq s \\
E\left[e(t) e^{T}(t)\right] & =R_{e} .
\end{aligned}
$$

The process disturbance $v$, on the other hand, can both be of noise character, affecting the state at every time instant, or occasionally occurring impulses. Any particularly common shape of the disturbance, like a step, a ramp or a sinusoid, can via the state equation (matrices 
$G$ and $A$ ) be constructed as the response of a driving impulse.

Our interest lies in obtaining reliable state estimates $\hat{x}$ under impulsive process disturbance, but we do not require the estimates in real time. That is, the estimate of the state at time $t, \hat{x}(t)$, can be obtained at a later time $N$. This is known as the smoothing problem in filtering theory.

In the next section we describe a deterministic framework for handling the detection and estimation of such disturbances. In Section 3 we describe how to formulate the case of impulsive process disturbances in a stochastic framework, and what methods and algorithms for detection and state estimation this leads to.

The approach we suggest in the present article is somewhere between these approaches. It is described in Section 4 and is based on convex optimization of a criterion that can be seen as a modification of either the deterministic or the stochastic formulation. It is inspired by the recent progress of sum-of-norms regularization in the statistical literature, [21], and is also related to contributions in the control community, [27].

\section{A Deterministic Framework for State Estima- tion Under Impulsive Process Disturbances}

The assumption of an impulsive process disturbance implies that $v(t)$ is a vector that is zero for most $t$ and takes unknown, nonzero values at a few unknown time instances. At this point, consider a certain time interval $[1, N]$ where $v(t)$ is nonzero for precisely one time instant. The linear state space model with an impulsive disturbance occurring in the process model is then:

$$
\begin{aligned}
x(t+1) & =A x(t)+B u(t)+G v(t) \\
y(t) & =C x(t)+e(t), \\
v(t) & = \begin{cases}v^{*} & t=t^{*} \\
0 & t \neq t^{*} .\end{cases}
\end{aligned}
$$

Assume $e(t) \sim N\left(0, R_{e}\right)$ and $x(1)=0$ for simplicity and assume that the model parameters $A, B, G, C$, are known.

\subsection{The Approach by Willsky and Jones}

The state estimation problem then boils down to estimating $t^{*}$ and $v^{*}$ from input-output data $y(t), u(t), t=$ $1, \ldots, N$. This can be solved as a least squares problem as follows: Suppose that $v\left(t^{\prime}\right) \neq 0$ and $v(t)=0, t=$ $1, \ldots, t^{\prime}-1, t^{\prime}+1, \ldots, N-1$. The data for $t \in\left[t^{\prime}+\right.$ $1, \ldots, N]$ can then be used to compute the least-squares estimate

$$
\hat{v}\left(t^{\prime}\right)=\left(\Phi^{T} R_{e}^{-1} \Phi\right)^{-1} \Phi^{T} R_{e}^{-1} Y,
$$

with

$Y \triangleq\left[\begin{array}{c}y\left(t^{\prime}+1\right)-C \sum_{t=1}^{t^{\prime}} A^{t^{\prime}-t} B u(t) \\ y\left(t^{\prime}+2\right)-C \sum_{t=1}^{t^{\prime}+1} A^{t^{\prime}+1-t} B u(t) \\ \vdots \\ y(N)-C \sum_{t=t^{\prime}}^{N-1} A^{N-1-t} B u(t)\end{array}\right], \Phi \triangleq\left[\begin{array}{c}C G \\ C A G \\ \vdots \\ C A^{N-1-t^{\prime}} G\end{array}\right]$,

and its covariance matrix

$$
P\left(t^{\prime}\right)=\left(\Phi^{T} R_{e}^{-1} \Phi\right)^{-1}
$$

The significance of the least squares estimate can be formed as

$$
\ell\left(t^{\prime}\right)=\hat{v}^{T}\left(t^{\prime}\right) P^{-1}\left(t^{\prime}\right) \hat{v}\left(t^{\prime}\right) .
$$

This variable has a $\chi^{2}(d)(d=\operatorname{dim} v)$ distribution if the true value of $v\left(t^{\prime}\right)=0$. Pick as estimate of the jump time $t^{*}$ that value of $t^{\prime}$ that maximizes this significance $\ell\left(t^{\prime}\right)$.

If we do not know for sure that there is a jump in the interval $[1, N-1]$, we can decide if the indicated jump is a significant indication by the test

$$
\ell\left(t^{\prime}\right)>T
$$

where $T$ is a suitably chosen significance level, according to the $\chi^{2}(d)$ distribution.

This is the well known Willsky-Jones (WJ) Generalized Likelihood Ratio (GLR) approach, [36]. See also Section 9.3 in [17] and the related approach [16].

Remark 1 In [36] also a stochastic process disturbance and a stochastic initial value $x(1)$ are allowed. Then a Kalman filter for the nominal case (discarding the impulsive disturbance component in $v$ ) is constructed, and the contribution of a deterministic $v$ to the innovations (residuals) $\varepsilon$ from this filter is analyzed. That is, $\varepsilon(t)$ plays the role of $y(t)$ in the calculations above.

Another description of the WJ-approach is as follows: Let

$$
W(v(\cdot))=\sum_{t=2}^{N}\left\|R_{e}^{-1 / 2}(y(t)-C x(t))\right\|_{2}^{2},
$$

where $x(t+1)=A x(t)+B u(t)+G v(t), x(1)=0$. Solve

$$
\begin{aligned}
& \min _{v(k), k=1, \ldots, N-1} W(v(\cdot)) \\
& \text { s.t. }\|V\|_{0}=1 ; V=\left[\|v(1)\|_{2}, \ldots,\|v(N-1)\|_{2}\right] .
\end{aligned}
$$

Here $\|V\|_{0}$ is the $\ell_{0}$ norm, i.e., the cardinality (number of nonzero elements) of the vector $V$. Due to the constraint $\|V\|_{0}=1,(6)$ will result in an estimate for $v(t)$ which is 
nonzero for precisely one time instant. Hence, to solve the non-convex problem (6),

$$
\begin{aligned}
& \min _{v(k), k=1, \ldots, N-1} W(v(\cdot)) \\
& \text { s.t. } \quad v(t)=0, t=1, \ldots, t^{\prime}-1, t^{\prime}+1, \ldots, N-1,
\end{aligned}
$$

can be solved for $t^{\prime}=1, \ldots, N-1$. The $v$-estimate giving the smallest objective value clearly also solves (6). It can further be shown that this estimate is equivalent to that of the WJ-approach. The equivalence follows from the following lemma.

Lemma 2 For any $t^{\prime} \in[1, \ldots, N-1]$, the criterion $(7)$ has the objective value

$$
\sum_{t=2}^{N}\left\|R_{e}^{-1 / 2}\left(y(t)-C \sum_{s=1}^{t-1} A^{s-1} B u(t-s)\right)\right\|_{2}^{2}-\ell\left(t^{\prime}\right)
$$

at the optimum. Hence, the $t^{\prime}$ that gives the smallest objective value of (7) also maximizes $\ell$ in (5a).

PROOF. This is a well known property of least squares estimation: First rewrite (7) as a least squares problem by substituting the constraint into the objective. Then, using the same definitions for $Y$ and $\Phi$ as used in (3), (7) takes the form

$$
\begin{aligned}
& \min _{v\left(t^{\prime}\right)}\left(Y-\Phi v\left(t^{\prime}\right)\right)^{T} R_{e}^{-1}\left(Y-\Phi v\left(t^{\prime}\right)\right) \\
& +\sum_{t=2}^{t^{\prime}}\left\|R_{e}^{-1 / 2}\left(y(t)-C \sum_{s=1}^{t-1} A^{s-1} B u(t-s)\right)\right\|_{2}^{2} \\
& =\min _{v\left(t^{\prime}\right)}\left\|R_{e}^{-1 / 2}\left(\left(\Phi^{T} R_{e}^{-1} \Phi\right)^{-1} \Phi^{T} R_{e}^{-1} Y-v\left(t^{\prime}\right)\right)\right\|_{2}^{2} \\
& \quad-Y^{T} R_{e}^{-1} \Phi\left(\Phi^{T} R_{e}^{-1} \Phi\right)^{-1} \Phi^{T} R_{e}^{-1} Y \\
& +\sum_{t=2}^{N}\left\|R_{e}^{-1 / 2}\left(y(t)-C \sum_{s=1}^{t-1} A^{s-1} B u(t-s)\right)\right\|_{2}^{2} \\
& =\min _{v\left(t^{\prime}\right)}\left\|R_{e}^{-1 / 2}\left(\left(\Phi^{T} R_{e}^{-1} \Phi\right)^{-1} \Phi^{T} R_{e}^{-1} Y-v\left(t^{\prime}\right)\right)\right\|_{2}^{2} \\
& \quad+\sum_{t=2}^{N}\left\|R_{e}^{-1 / 2}\left(y(t)-C \sum_{s=1}^{t-1} A^{s-1} B u(t-s)\right)\right\|_{2}^{2}-\ell\left(t^{\prime}\right) .
\end{aligned}
$$

The objective is now trivially minimized by

$$
v\left(t^{\prime}\right)=\left(\Phi^{T} R_{e}^{-1} \Phi\right)^{-1} \Phi^{T} R_{e}^{-1} Y
$$

and then takes the value (8).

If $n$ jumps are allowed, the only difference in the formulation (6) is that $\|V\|_{0}=n$ instead (which for larger $n$ will be combinatorially forbidding). If the number of jumps is not known, a trade-off can be formulated as

$$
\begin{aligned}
& \min _{v(k), k=1, \ldots, N-1} \sum_{t=1}^{N}\left\|R_{e}^{-1 / 2}(y(t)-C x(t))\right\|_{2}^{2}+\lambda\|V\|_{0} \\
& \text { s.t. } x(t+1)=A x(t)+B u(t)+G v(t) ; x(1)=0 .
\end{aligned}
$$

The parameter $\lambda>0$ sets the trade-off between the number of jumps and the fit to the measurements $y$. (10) is a combinatorial non-convex optimization problem and in general, $2^{N-1}$ constrained least squares problems have to be solved to find the minimizing $v$.

Remark 3 The case with several jumps is handled in [36] by performing the above search for one jump, recursively over sliding windows of length $F$. Once a jump has been indicated, its influence on $y$ is removed (i.e., the residuals $\varepsilon$ are calculated) before the search for the next jump is continued.

\subsection{The CUSUM Test}

The key element in the Willsky-Jones GLR approach is to find a deterministic component $v$ in a stochastic signal $y(t+1)-C \sum_{s=1}^{t} A^{t-s} B u(s)$ (or the Kalman filter residual $\varepsilon$ ). The WJ approach can be seen as an optimal matched filter for this deterministic signal. A more simplistic approach would be to try to detect nonzero deterministic components in the theoretically zero-mean sequence $y$ using a change detection method. This is the approach taken in the CUSUM test (Cumulative Sum [29], see also Algorithm 1).

\section{Algorithm 1 CUSUM}

Set $g(1)=0$. For a chosen $\gamma_{c}$ and $h$, the time of a change in the signal $r(t)$ is estimated by observing when

$$
g(t+1)=\max \left(g(t)+r(t)-\gamma_{c}, 0\right)
$$

exceeds the threshold $h$. After a change has been detected, $g$ is reset to zero and the last $t$ for which $g(t)=0$ taken as an estimate of the change time.

The CUSUM algorithm detects a change in the mean of the signal by checking when the test statistic $g$ (see Algorithm 1) exceeds some threshold $h$. Since $g$ is built up by summing consecutive signal values, a large $g$ is an indication that the mean has changed and has been considerably larger than zero for some time. To also detect that the mean has changed to something smaller than zero, $r(t)$ in (11) should be replaced by $-r(t)$.

Note that the CUSUM algorithm is a change detection algorithm and seeks the time of a change while WillskyJones GLR approach directly estimates $v\left(t^{*}\right)$. Of course, 
having estimated the time of the change, say $t^{\prime}$, an estimate for $v\left(t^{\prime}\right)$ can be found through

$$
\begin{aligned}
& \min _{v(k), k=1, \ldots, N-1} W(v(\cdot)) \\
& \text { s.t. } v(t)=0, t=1, \ldots, t^{\prime}-1, t^{\prime}+1, \ldots, N-1 .
\end{aligned}
$$

\section{A Stochastic Framework for State Estimation Under Impulsive Process Disturbances}

The standard linear state space model with stochastic disturbances is well known to be

$$
\begin{aligned}
x(t+1) & =A x(t)+B u(t)+G v(t) \\
y(t) & =C x(t)+e(t) .
\end{aligned}
$$

Here, $v$ and $e$ are white noises: sequences of independent random vectors

$$
\begin{aligned}
E[v(t)] & =0, \quad E[e(t)]=0, \quad \forall t \\
E\left[v(t) e^{T}(s)\right] & =0, \quad \forall t, s \\
E\left[v(t) v^{T}(s)\right] & =0, \quad E\left[e(t) e^{T}(s)\right]=0, \quad t \neq s \\
E\left[v(t) v^{T}(t)\right] & =R_{v}, \quad E\left[e(t) e^{T}(t)\right]=R_{e} .
\end{aligned}
$$

The independence of the noise sequences is required in order to make $x$ a state or a Markov process.

The model (13) with the process disturbance $v$ being Gaussian is a standard model for control applications. $v$ then represents the combined effect of all those nonmeasurable inputs that in addition to $u$ affect the states. This is the common model used both for state estimation and in Linear-Quadratic-Gaussian (LQG) control.

The state smoothing problem is well known in this Gaussian case as the classical Kalman smoothing problem, e.g., [20]. Viewing $x(t)$ as a function of $u$ and $v$, the smoothed state is the solution to the quadratic minimization problem

$$
\begin{aligned}
\min _{x(1), v(t), 1 \leq t \leq N-1} & \sum_{t=1}^{N}\left\|R_{e}^{-1 / 2}(y(t)-C x(t))\right\|_{2}^{2} \\
& +\sum_{t=1}^{N-1}\left\|R_{v}^{-1 / 2} v(t)\right\|_{2}^{2}, \\
\text { s.t. } \quad x(t+1) & =A x(t)+B u(t)+G v(t) .
\end{aligned}
$$

This is also the maximum likelihood estimate and gives the conditional mean of $x(t)$ given the observations. It is a pure least squares problem, and the solution is usually given in various recursive filter forms, see e.g., [25].

Since $x(t)$ is a given function of $x(1), v(t)$ and the known sequence $u(t)$, it may seem natural to do the minimiza- tion directly over $x(t)$, i.e., to write

$$
\begin{aligned}
& \min _{x(t), 1 \leq t \leq N} \sum_{t=1}^{N}\left\|R_{e}^{-1 / 2}(y(t)-C x(t))\right\|_{2}^{2} \\
& +\sum_{t=1}^{N-1}\left\|R_{v}^{-1 / 2} G^{\dagger}(x(t+1)-A x(t)-B u(t))\right\|_{2}^{2}
\end{aligned}
$$

where $G^{\dagger}$ is the pseudo inverse of $G$. However, if $G$ is not full rank, nothing constrains the state evolution in the null space of $G$, so (15a) must be complemented with the constraint

$$
G^{\perp}(x(t+1)-A x(t)-B u(t))=0
$$

where $G^{\perp}$ is the projection onto the null-space of $G$,

$$
G^{\perp} \triangleq I-G G^{\dagger}
$$

However, since several approaches can be interpreted as explicit methods to estimate $v(t)$, we shall adhere to the (equivalent) formulation (14).

\subsection{Sparse Process Disturbance}

The situation of current interest is that the process disturbance is zero most of the time, and assumes unknown nonzero values at unknown time instants. It is convenient to capture this by the distribution ( $c f$. eq (2.10)$(2.11)$ in [24].)

$$
\begin{gathered}
v(t)=\delta(t) \eta(t) \\
\text { where } \\
\delta(t)= \begin{cases}0 & \text { with probability } 1-\mu \\
1 & \text { with probability } \mu\end{cases} \\
\eta(t) \sim N(0, Q) .
\end{gathered}
$$

This makes $R_{v}=\mu Q$.

\subsection{State Estimates Based on a Stochastic Framework}

The best linear solution: Even with the nonGaussian noise (17), the process disturbance is still white, which means the Kalman-smoother (14) still gives the best linear estimate. This implies that among all estimates $\hat{x}$ that are linear functions of $x(1), y$ and $u$, the Kalman-smoother residual has the smallest variance.

The clairvoyant estimator: If the jump times, the times when $\delta(t)=1$ in (17a), are known, the random variable $v(t)$ is Gaussian (with time-varying covariance matrix $\left.R_{v}(t)=\delta(t) Q\right)$. This means that the Kalman smoother (14) is not only the best linear estimator, but also gives the conditional mean and the estimate with the smallest variance among all possible ones. 
Filter banks: If $\delta(t)$ is not known, we could hypothesize in each time step that it is either 0 or 1 . This leads to a large bank $\left(2^{N-1}\right)$ of Kalman filters, which constitute the optimal solution. The posterior probability of each filter estimate can be determined, and the optimal estimate is a weighted sum of the state estimates from each filter. In practice, the number of filters in the bank must be limited, and there are two main options (see Chapter 10 in [18]):

- Merging trajectories of different $\delta(t)$ sequences. This includes the well-known IMM (Interactive Multiple Model) filter, see [4] and e.g., [23] for a smoothing formulation.

- Pruning, where unlikely sequences are deleted from the filter bank.

Remark 4 The Willsky-Jones GLR approach can also be given an interpretation as a filter bank, see e.g., [36,35].

Particle filters: Since (13) and (17) form a linear model with non-Gaussian noise, nonlinear filtering/smoothing techniques based on particle filtering ([13], see also [1] for a relevant contribution) can also be applied. This solution means that a set of new particles are created at each time instant, corresponding to the possibility that $\delta(t)$ is nonzero, and these will then survive if supported by future measurement. This is in spirit very much like Kalman filter banks. Experience shows that the Kalman filter bank is quite efficient to explore multiple hypotheses. The standard SIR (Sequential Importance Resampling) particle filter works well for low and moderate SNRs (Signal-to-Noise Ratio), but starts to degenerate due to depletion for very large SNRs. This can be mitigated with other proposal distributions and resampling schemes, but still the particle filter/smoother cannot compete with a good implementation of a Kalman filter/smoother bank.

\section{The Proposed Method: State Smoothing by Sum-of-Norms Regularization}

We may regard (10) or the filter banks as ideal methods for state estimation in linear state space models where impulsive disturbances occur in the process model. The catch is that these are both computationally forbidding. One way to obtain a computationally feasible method is to follow the idea from compressed sensing [10,8], and replace the $\ell_{0}$-norm in (10) by the $\ell_{1}$ norm. That makes the optimization problem convex, at the same time as several of the good features of the $\ell_{0}$-norm are retained, $[10,8]$, e.g., sparsity (see e.g., [19, pp. 70-71]).

\subsection{Sum-of-Norms Regularization}

Consider

$$
\begin{gathered}
\min _{v(k), k=1, \ldots, N-1} \sum_{t=1}^{N}\left\|R_{e}^{-1 / 2}(y(t)-C x(t))\right\|_{2}^{2}+\lambda\|V\|_{0} \\
\text { s.t. } x(t+1)=A x(t)+B u(t)+G v(t), x(1)=0, \\
V=\left[\left\|Q^{-1 / 2} v(1)\right\|_{p}, \ldots,\left\|Q^{-1 / 2} v(N-1)\right\|_{p}\right]
\end{gathered}
$$

which is the same as (10) up to a scaling of $V$. Since the $\ell_{0}$-norm is invariant to scalings, it is easily verified that (10) and (18) give the same $v$-estimates. Now, using the $\ell_{1}$ instead of the $\ell_{0}$-norm on $V$ in (18) and relaxing the constraint $x(1)=0$ gives the optimization problem

$$
\begin{array}{r}
\min _{x(1), v(t), 1 \leq t \leq N-1} \sum_{t=1}^{N}\left\|R_{e}^{-1 / 2}(y(t)-C x(t))\right\|_{2}^{2} \\
\quad+\lambda \sum_{t=1}^{N-1}\left\|Q^{-1 / 2} v(t)\right\|_{p} \\
\text { s.t. } x(t+1)=A x(t)+B u(t)+G v(t) .
\end{array}
$$

The parameter $\lambda$, as in (10), is a positive constant that is used to control the trade-off between the fit to the observations $y(t)$ (the first term) and the number of nonzero $v(t)$ (the second term). The weighted $p$-norm could be any $\ell_{p}$-norm, like $\ell_{1}$ or $\ell_{2}$. It is however crucial for sparse solutions that the second term in (19a) is a sum of norms, and not a sum of squared norms.

While this criterion is grown from the deterministic approach, it is interesting to see its connection with the stochastic criterion (15a). The expression (15a) can be seen as the criterion of fit

$$
\min _{x(1), v(t), 1 \leq t \leq N-1} \sum_{t=1}^{N}\left\|R_{e}^{-1 / 2}(y(t)-C x(t))\right\|_{2}^{2}
$$

regularized by the quadratic term

$$
\sum_{t=1}^{N-1}\left\|R_{v}^{-1 / 2} v(t)\right\|_{2}^{2}
$$

in order to curb the flexibility of $v$. Our criterion (19) just replaces this quadratic regularization with the sumof-norms

$$
\sum_{t=1}^{N-1}\left\|Q^{-1 / 2} v(t)\right\|_{p}
$$

When the $p$-norm of $v(t)$ is taken to be the $\ell_{1}$ norm, i.e., $\|z\|_{1}=\sum_{i=1}^{n_{z}}\left|z_{i}\right|$, the regularization in (19a) is a standard $\ell_{1}$ regularization of the least-squares criterion. Such regularization has been very popular recently, e.g., 
in the much used Lasso method, [34] or compressed sensing $[10,8]$.

There are two key reasons why the criterion (19a) is attractive:

- It is a convex optimization problem, so the global solution can be computed efficiently. In fact, its special structure allows it to be solved in $O(N)$ operations, so it is quite practical to solve it for a range of values of $\lambda$, even for large values of $N$.

- The sum-of-norms form of the regularization favors sparse solutions where many (depending on $\lambda$ ) of the regularized variables come out as exactly zero in the solution. In this case, this implies that many of the estimates of $v(t)$ become zero (with the number of $v(t)$ 's becoming zero controlled roughly by $\lambda$ ).

A third advantage is that

- It is easy to include realistic state constraints without destroying convexity.

The downside of using an $\ell_{1}$-norm instead of the $\ell_{0}$-norm is that the $\ell_{1}$-norm penalizes the size of the regularized variable and not only if it is nonzero or not, like the $\ell_{0^{-}}$ norm. The regularized variable $\left(\left\|Q^{-1 / 2} v(t)\right\|_{p}\right.$ in (19a)) will therefore be biased toward zero. We return to this issue in Section 5.3.

We should comment on the difference between using $p=1$ in (19a), giving an $\ell_{1}$ regularization on $Q^{-1 / 2} v(t)$, and some other type of sum-of-norms regularization, such as sum-of-Euclidean norms (which gives an $\ell_{1}$ regularization on $\left.\left[\left\|Q^{-1 / 2} v(1)\right\|_{2}, \ldots,\left\|Q^{-1 / 2} v(N-1)\right\|_{2}\right]\right)$. With $\ell_{1}$ regularization, we obtain an estimate of $Q^{-1 / 2} v(t)$ having many of its components equal to zero. When we use sum-of-norms regularization with $p>1$, the whole estimated vector $Q^{-1 / 2} v(t)$ often becomes zero; but when it is nonzero, typically all its components are nonzero. Note however that both $p=1$ and $p>1$ give an $\ell_{1}$-regularization on the vector $\left[\left\|Q^{-1 / 2} v(1)\right\|_{p}, \ldots,\left\|Q^{-1 / 2} v(N-1)\right\|_{p}\right]$. In a statistical linear regression framework, sum-of-norms regularization with $p>1$ is called group-lasso [37], since it results in estimates in which many groups of variables are zero.

Remark 5 A criterion (19a) handles the process disturbance as described in (17) well. In some situations it may however be more accurate to assume a Gaussian noise component in the process disturbance as well, i.e.,

$$
\begin{aligned}
x(t+1) & =A x(t)+B u(t)+G v(t)+H w(t) \\
y(t) & =C x(t)+e(t)
\end{aligned}
$$

with $w \sim N(0, S)$ and $v$ as defined in (17). It is then motivated to use a criterion

$$
\begin{aligned}
& \min _{\substack{x(1), v(t), w(t) \\
1 \leq t \leq N-1}} \sum_{t=1}^{N}\left\|R_{e}^{-1 / 2}(y(t)-C x(t))\right\|_{2}^{2} \\
& \quad+\sum_{t=1}^{N-1} \lambda\left\|Q^{-1 / 2} v(t)\right\|_{p}+\left\|S^{-1 / 2} w(t)\right\|_{2}^{2} \\
& \text { s.t. } \quad x(t+1)=A x(t)+B u(t)+G v(t)+H w(t)
\end{aligned}
$$

rather than (19a).

\section{Choice of Regularization Parameter $\lambda$}

\subsection{Regularization Path and Critical Parameter Value}

The estimated sequence $v(t)$ as a function of the regularization parameter $\lambda$ is called the regularization path for the problem. Roughly, larger values of $\lambda$ correspond to estimated $x(t)$ with worse fit, but an estimate of $v(t)$ with many zero elements. A basic result from convex analysis (see Remark 2 in [28], also $c f$. pp. 277-278 in [7]) tells us that there is a value $\lambda^{\max }$ for which the estimated $v(t)$ is identically zero if and only if $\lambda \geq \lambda^{\max }$. In other words, $\lambda^{\max }$ gives the threshold above which the estimated $v(t)=0, t=1, \ldots, N-1$. The critical parameter value $\lambda^{\max }$ is very useful in practice, since it gives a very good starting point in finding a suitable value of $\lambda$.

Proposition 6 The Critical Value $\lambda^{\max }$

Introduce $\varepsilon_{t}$ for the (process) noise free scaled residual

$$
\varepsilon_{t} \triangleq R_{e}^{-1 / 2}\left(y(t)-C\left(\sum_{r=1}^{t-1} A^{t-r-1} B u(r)+A^{t-1} x(1)\right)\right)
$$

and take $\bar{\varepsilon}_{t}$ to be $\varepsilon_{t}$ evaluated at

$$
x(1)=\underset{x(1)}{\arg \min } \sum_{t=1}^{N}\left\|\varepsilon_{t}\right\|_{2}^{2} .
$$

We can then express $\lambda^{\max }$ as

$$
\lambda^{\max }=\max _{k=1, \ldots, N-1}\left\|2 \sum_{t=k+1}^{N}\left(R_{e}^{-1 / 2} C A^{t-k-1} G Q^{1 / 2}\right)^{T} \bar{\varepsilon}_{t}\right\|_{q}
$$

with $\|\cdot\|_{q}$ the dual norm $(1 / p+1 / q=1)$ associated with $\|\cdot\|_{p}$ used in (19a).

The proof is given in the appendix. 


\subsection{Signal-to-Noise Ratio and the Choice of $\lambda$}

The regularization parameter $\lambda$ controls the trade-off between the fit of $x$ to observations and the amount of process disturbance $v$ that can be used for that fit. This reflects the signal-to-noise ratio of the data: The more signal influence (process disturbances $v$ ), the more important the second term of (19a) and vice versa.

The criterion (19a) is not homogeneous. Since the second term is a norm and the first one is a squared norm, the solution will change if we rescale the problem by multiplying all signals by a scalar.

A criterion with $\lambda$ based on a fraction of $\lambda^{\max }$,

$$
\begin{aligned}
\min \sum \| R_{e}^{-1 / 2}(y(t) & -C x(t)) \|_{2}^{2} \\
& +\beta \lambda^{\max } \sum\left\|Q^{-1 / 2} v(t)\right\|_{p},
\end{aligned}
$$

will handle this, giving a criterion that is invariant to scaling: If $R_{e}$ and $Q$ are multiplied by the same scalar, the minimization problem is not affected. Letting the choice of $\lambda$ be based on $\lambda^{\max }$ is thus a sound principle. But note that (27) is also invariant to all scaling in the scalar case $\left(y, v, R_{e}, Q\right.$ being scalars): $R_{e}$ and $Q$ can be changed to any positive numbers, without affecting the solution if $\beta$ is a given constant. To allow the second term to have more influence at high signal-to-noise ratios, we propose the following basic choice of regularization parameter:

$$
\lambda=\frac{1}{10} \sqrt{\frac{\left\|R_{e}\right\|}{\|Q\|}} \lambda^{\max }
$$

The choice of the scaling $1 / 10$ is admittedly ad hoc, but governed by the fact that if the amplitude signal-to-noise ratio is less that 0.1 , no jumps $v$ will be indicated by criterion (19). This is a reasonable choice, since at such a low signal-to-noise ratio the risk of false jump detections is very high.

Such signal-to-noise tuning is present in all state estimation algorithms. For example the Willsky-Jones method (5) requires the noise level $R_{e}$ to determine the covariance matrix $P$ and knowledge of typical jump sizes to design a proper significance level $T$ to minimize the risk of false detections. The Kalman filter, the particle filter, the IMM, all require such knowledge to be properly tuned.

\subsection{Iterative Refinement}

To (possibly) get even more zeros in the estimate of $v(t)$, with no or small increase in the fitting term, iterative reweighting can be used [9]. We modify the regularization term in (19a) and consider

$$
\begin{aligned}
\min _{x(1), v(t), 1 \leq t \leq N-1} & \sum_{t=1}^{N}\left\|R_{e}^{-1 / 2}(y(t)-C x(t))\right\|_{2}^{2} \\
& +\lambda \sum_{t=1}^{N-1} \alpha(t)\left\|Q^{-1 / 2} v(t)\right\|_{p}
\end{aligned}
$$

where $\alpha(1), \ldots, \alpha(N-1)$ are positive weights used to vary the regularization over time. Iterative refinement proceeds as follows. We start with all weights equal to one i.e., $\alpha^{(0)}(t)=1$. Then for $i=0,1, \ldots$ we carry out the following iteration until convergence (which is typically in just a few steps).

(1) Find the state estimate.

Compute the optimal $v^{(i)}(t)$ using (29) with the weighted regularization using weights $\alpha^{(i)}$.

(2) Update the weights.

For $t=1, \ldots, N-1$, set $\alpha^{(i+1)}(t)=1 /(\epsilon+$ $\left.\left\|Q^{-1 / 2} v^{(i)}(t)\right\|_{p}\right)$. In this situation the value of $\lambda$ can be reduced, to obtain better estimates of the jump size at the jump instances.

Here $\epsilon$ is a positive parameter that sets the maximum weight that can occur.

As already stated, several of the good features of the $\ell_{0^{-}}$ norm are retained when replaced by the $\ell_{1}$-norm. However, the downside is that the $\ell_{1}$-norm penalizes the size of the regularized variable and not only if it is nonzero or not, like the $\ell_{0}$-norm. The regularized variable will therefore be biased toward zero. To get rid of this bias, one final step is useful. From our final estimate of $v(t)$, we simply define the set of times $\mathcal{T}$ at which an estimated load disturbance occurs (i.e., $\mathcal{T}=\{t \mid v(t) \neq 0\}$ ) and carry out a final optimization over just $v(t), t \in \mathcal{T}$,

$$
\begin{gathered}
\min _{x(1), v(t), 1 \leq t \leq N-1} \sum_{t=1}^{N}\left\|R_{e}^{-1 / 2}(y(t)-C x(t))\right\|_{2}^{2} \\
\text { s.t. } x(t+1)=A x(t)+B u(t)+G v(t) \\
v(t)=0 \text { if } t \notin \mathcal{T} .
\end{gathered}
$$

\section{Summary of the Algorithm - STATESON}

The algorithm is summarized in Algorithm 2.

Algorithm 2 State Estimation by Sum-of-Norms Regularization (STATESON)

Given $A, B, C, G, R_{e}, Q$ and $\{(y(t), u(t))\}_{t=1}^{N}$. Let $\epsilon$ be a positive parameter, set $\alpha^{(0)}(t)=1$ for $t=1, \ldots, N-1$ and $i=0$. Then, for a given $\lambda$ (e.g., determined as (28)) 
(1) Compute the optimal $v^{(i)}(t)$ using (29) with $\alpha=$ $\alpha^{(i)}$.

(2) $\operatorname{Set} \alpha^{(i+1)}(t)=1 /\left(\epsilon+\left\|Q^{-1 / 2} v^{(i)}(t)\right\|_{p}\right)$. Possibly also reduce $\lambda$ at this step.

(3) If convergence, go to the next step, otherwise set $i=i+1$ and return to step 1 .

(4) Compute a final estimate of $v(t)$ using (30).

Remark 7 If the jump covariance $Q$ in (17) is known or can be given a good value, the final optimization step (step (4) in Algorithm 2) could be replaced by a Kalman smoother with the time-varying process disturbance

$$
R_{v}(t)= \begin{cases}0 & \text { fort's such that } v(t)=0 \\ Q & \text { otherwise }\end{cases}
$$

It should be noticed that if the correct jump-times and $Q$ have been found, this is actually optimal in the sense that no other smoother (linear or nonlinear) can achieve an unbiased estimate with a lower error covariance. Since everything is Gaussian (time varying covariance), this follows from the optimality of the Kalman smoother.

\section{Solution Algorithms and Software}

Many standard methods of convex optimization can be used to solve the problem (19). Systems such as CVX $[15,14]$ or YALMIP [26] can readily handle the sumof-norms regularization, by converting the problem to a cone problem and calling a standard interior-point method. For the special case when the $\ell_{1}$ norm is used as the regularization norm, more efficient special purpose algorithms and software can be used, such as 11_ls [22]. Recently many authors have developed fast first order methods for solving $\ell_{1}$ regularized problems, and these methods can be extended to handle the sum-of-norms regularization used here; see, for example, [32, §2.2]. Methods such as Alternating Direction Method of Multipliers (ADMM, [11,12], see also [6]), which are equivalent to other methods (such as Douglas-Rachford splitting) can also handle the sum-of-norms regularization and should be an attractive choice for large-scale problems. All of these methods have a complexity that scales linearly with $N$, and so can be applied to long data samples.

A CVX implementation of STATESON can be downloaded from http://www.rt.isy.liu.se/ ohlsson/ code.html.

\section{Numerical Illustration}

We will use a DC motor to illustrate how the disturbances can be handled. This is the same system as used in e.g., [24, pp. 95-97], ( $\left.T_{s}=0.1 \mathrm{~s}, \tau=0.286, \beta=40\right)$. The input is the applied torque and the output is the angle of the motor shaft. With states being angular velocity and angle, and a sampling time $T_{s}=0.1 \mathrm{~s}$, we obtain the discrete time model

$$
\begin{aligned}
x(t+1) & =\left[\begin{array}{ll}
0.7047 & 0 \\
0.08437 & 1
\end{array}\right] x(t)+\left[\begin{array}{c}
11.81 \\
0.6250
\end{array}\right](u(t)+v(t)) \\
s(t) & =\left[\begin{array}{ll}
0 & 1
\end{array}\right] x(t) \\
y(t) & =s(t)+e(t) .
\end{aligned}
$$

The transfer function from load disturbance $v$ to angle $y$ contains an integration, so a unit impulse in $v$ causes a change of level in $s$ of 4 units.

Example 8 Estimating Jump Times Using STATE$S O N$ and $W J$

Let us first study how well the STATESON algorithm can estimate the time of a single jump in $v$. We may think of the Willsky-Jones GLR approach (6) as a reference method, being the Maximum-Likelihood (ML) solution. We simulate (31) from $t=1$ to $t=100$ with $u \equiv 0$ (with no loss of generality, since the effect of the input is known anyway), $x(1)=0$ and

$$
v(t)= \begin{cases}0 & t \neq 55 \\ w & t=55\end{cases}
$$

with 10 different values of $w$ from 0.01 to 10 . The measurement noise is chosen as $e(t) \sim N(0,1)$. For each value of $w$ we generate 500 realizations of the measurement noise and estimate the jump time as

$$
\begin{aligned}
t_{W J} & =\arg \max _{t} \ell(t), \quad \ell(t) \text { defined by }(5 a), \\
t_{S T A T E S O N} & =\arg \max _{t} v(t) .
\end{aligned}
$$

Here $v(t)$ is defined as the solution to (19) with $R_{e}=$ $1, Q=1, p=2$ and $\lambda$ chosen as in (28). We then study the ensemble values of these estimates over the 500 measurement noise realizations. Histogram plots over the errors in estimated time are given in Figure 1. To illustrate how the accuracy of the jump time estimates depend on the jump size (i.e., the SNR) we plot in Figure 2 the square Root of the Mean Square Error (RMSE) of the estimate.

This example favors the Willsky-Jones approach, since it is devised to find one jump in the chosen interval. STATESON has no such information. For high SNR (jumps larger than 1) the WJ approach also outperforms STATESON. This is expected due to the asymptotic (errors tending to zero) optimality of the ML method. What is surprising is that STATESON does so well, and even outperforms WJ for small (jumps less than 1) SNRs.

If there is a possibility of several jumps in the signal, the situation is a bit more complex for the WJ approach. 

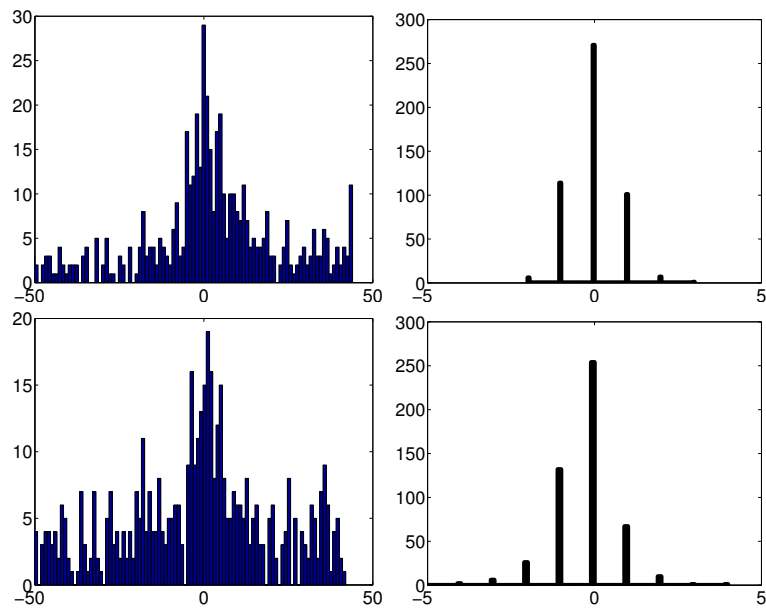

Fig. 1. Histogram over the errors in jump time for $w=0.1$ (left) and $w=1$ (right). Top: The Willsky Jones method. Bottom: The STATESON method.

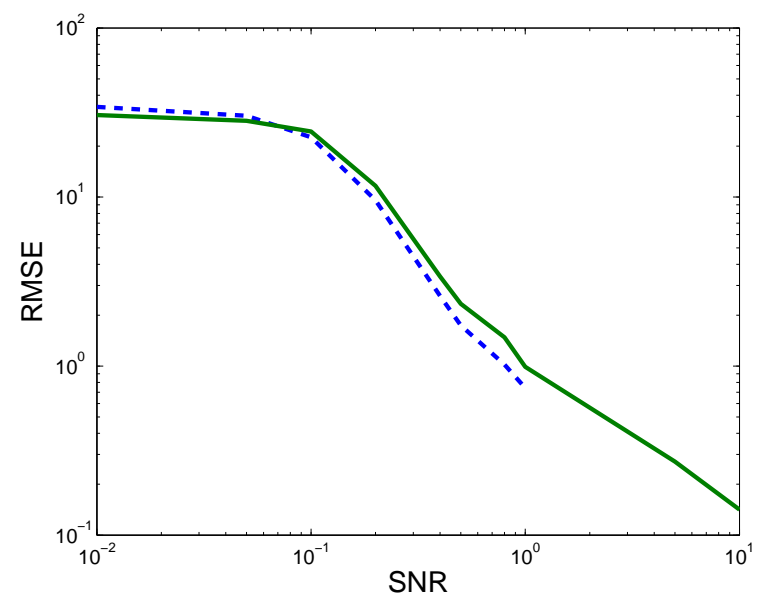

Fig. 2. Log-log diagram of the RMS error of the jump time estimate as a function of the SNR. Dashed line: Willsky-Jones. Solid line: STATESON. For the two highest SNRs the Willsky-Jones error is zero.

Then a sliding window must be applied as described in Remark 3. The resulting algorithm then has two important design variables: the threshold $T$ in (5b) and the window length $F$. Experimentation shows that the performance could be quite sensitive to these two variables.

We now turn to the problem of not primarily estimating jump times, but finding good smoothed state estimates.

Example 9 State Estimates by WJ and STATESON

We return to the system (31) in Example 8. Two jumps occur:

$$
\left\{\begin{array}{l}
v(49)=1 \\
v(55)=-1 \\
v(t)=0 \quad \text { otherwise }
\end{array}\right.
$$

The initial condition is fixed to $x(1)=0$, the measurement noise is e $(t) \sim N(0,1)$ and the system simulated for $t=1$ to 100. A typical realization of $y$ is shown in Figure 3. The state is estimated by the Willsky-Jones method

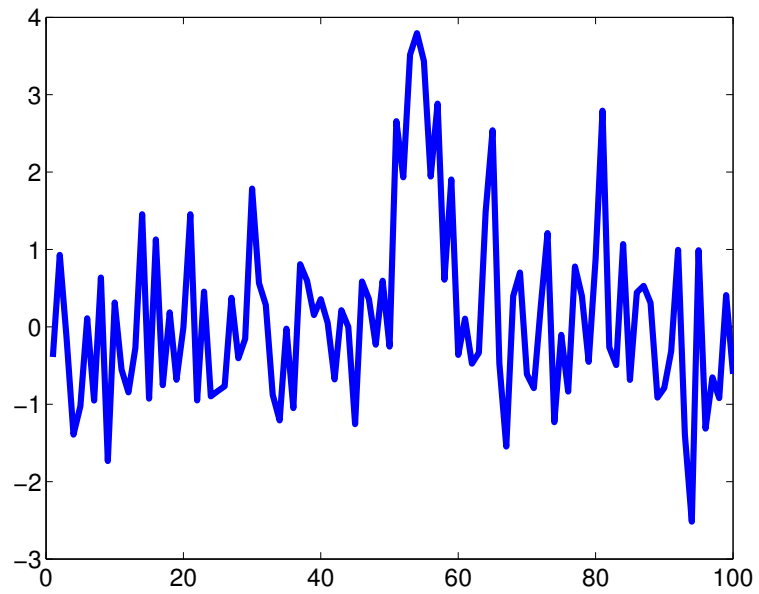

Fig. 3. A typical realization of $y$ in Example 9.

using a sliding window of length 5 and a threshold of 20. (See Remark 2.2 and (5b)). These design variables were optimized to give the best MSE over a Monte-Carlo study.

The state was also estimated by STATESON, Algorithm 2, with one iterative refinement. The parameter $\lambda$ was chosen as in (28) with $R_{e}=Q=1$, i.e., $\lambda=\lambda^{\max } / 10$ and it was further reduced by another factor 10 at the refinement iteration. The chosen norm was the Euclidean norm, i.e., $p=2$. The squared errors $\left|x_{2}(t)-\hat{x}_{2}(t)\right|^{2}$ as a function of $t$ averaged over 500 realizations of e are shown in Figure 4. A histogram plot over the 500 realizations of the norm $\sqrt{\sum_{t}\left|x_{2}(t)-\hat{x}_{2}(t)\right|^{2}}$ is shown in Figure 5.

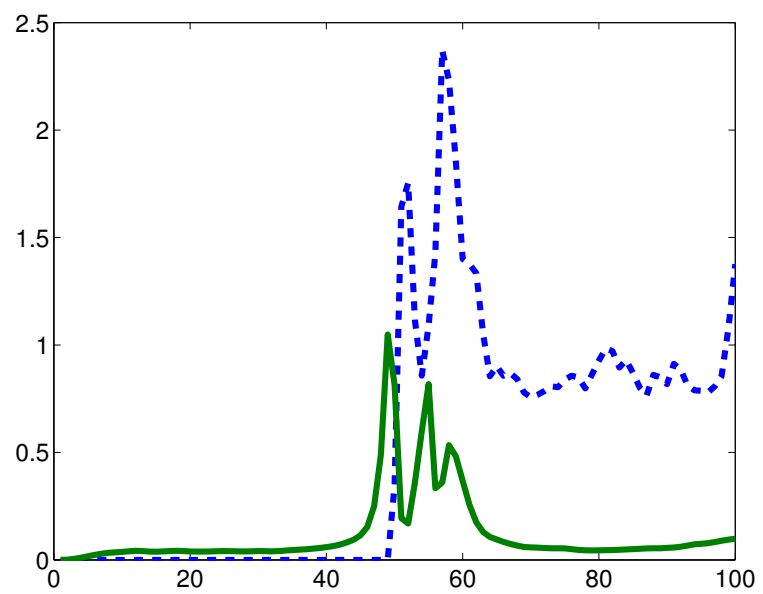

Fig. 4. The squared error of the second state estimate as a function of time, averaged over 500 measurement noise realizations. Dashed line: Willsky-Jones. Thick solid line: STATESON. 


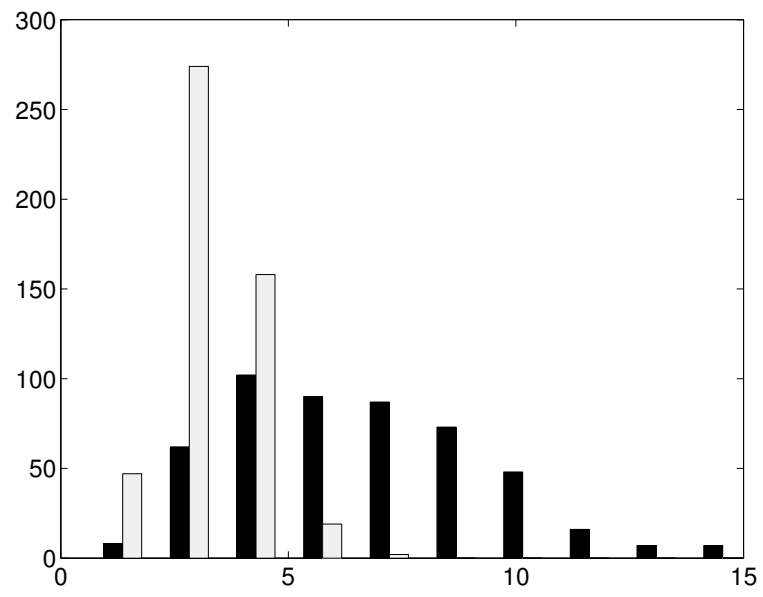

Fig. 5. Histogram plot for the error norms over 500 noise realizations. Light grey: STATESON. Black: Willsky-Jones. The $\mathrm{x}$-axis is the error norm, and the $\mathrm{y}$-axis shows the number of occurrences of the corresponding segment of error norm.

The example shows that the suggested STATESON method has a clear edge over the Willsky-Jones approach. This can probably be traced back to the fact that STATESON is a global method, while WillskyJones is a sliding, local method, in this application with possibly multiple jumps.

Let us also study the dependence of the signal-to-noise ratio and compare with the methods described in Sections 2 and 3.

Example 10 State Estimates Under Varying SNR and Multiple Jumps

Consider again the system given in (31). Let $v(t), t=$ $1, \ldots, 100$, be generated by (17) with $\mu=0.015, Q=10$, set $x(1)=0, u \equiv 0$ and vary $R_{e}$ to obtain different $S N R$ s. For each SNR-value, 500 Monte-Carlo runs (different $v$ and e realizations in each run) were carried out. State estimates were computed using:

(1) The lower bound according to the Clairvoyant smoother.

(2) Kalman smoother with the true $R_{e}$ and the change detection algorithm CUSUM (Cumulative Sum [29], see also Algorithm 1) to detect when to change between $R_{v}=0$ and $R_{v}=10$. CUSUM was applied to both the whitened innovations and the negative whitened innovations of a Kalman filter. A Kalman smoother was then applied with $R_{v}=10$ at the time instances of detected changes and $R_{v}=0$ otherwise. Several values for $h$ and $\gamma_{c}=1$ were tried out and $h=10$ and $\gamma_{c}=1$ gave the best performance and were used here.

(3) The proposed method STATESON according to Algorithm 2 with $\epsilon=10^{-4}$, two iterations, $\lambda$ as in (28) in the first iteration and a tenth of the that value in the second iteration.
(4) The Willsky and Jones GLR test, as described in Remark 3, with $T=25$ and $F=40$ for $R_{e} \geq 1$ and otherwise $T=85$ and $F=40$. Several $T$ and $F$-values were tested and these $T$ and $F$-values gave the best performance.

(5) A particle smoother with the true $R_{e}$ and a process disturbance distribution as in (17) with $\mu=0.015$ and $Q=10.1000$ particles were used.

(6) Conventional Kalman smoother with $R_{v}=\mu Q=$ 0.15 and the true $R_{e}$.

(7) An IMM smoother with two modes, the true $R_{e}$ for both and $R_{v}=0$ and 10 , respectively, with probabilities 0.985 and 0.015 . The IMM smoothing implementation of [33] was used.

The mean MSE $\left(1 / N \sum_{t}\left|x_{2}(t)-\hat{x}_{2}(t)\right|^{2}\right)$ taken over the 500 Monte-Carlo runs is shown for a number of $\sqrt{Q / R_{e}}$ values in Figure 6.

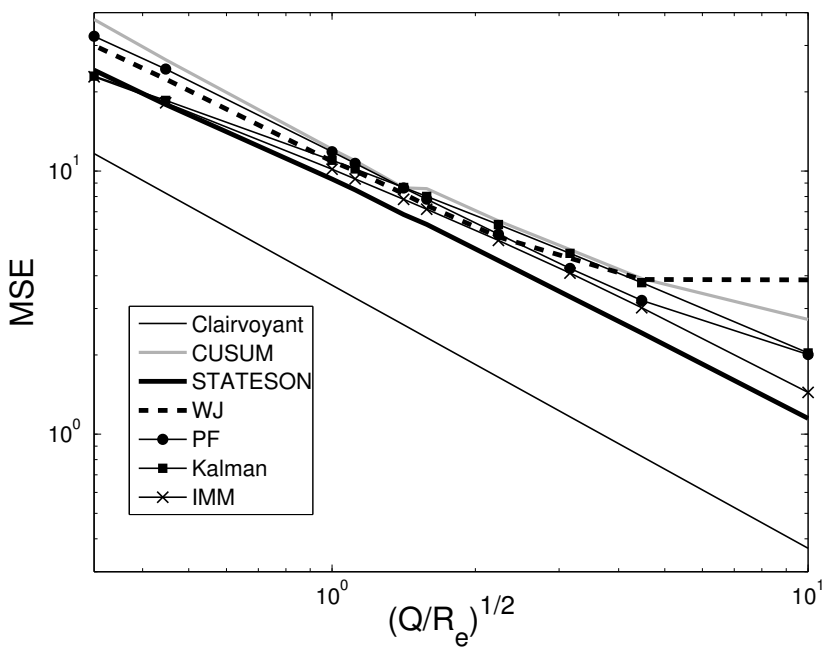

Fig. 6. A comparison between various methods for state estimation under impulsive process disturbances, as explained in Example 10. The $\mathrm{x}$-axis is the varying SNRs obtained by varying measurement disturbance variance $R_{e}$ and a constant jump size variance $Q=10$. The $\mathrm{y}$-axis is the mean square error of the second state variable over all the 100 time samples. 7 different methods are tested, as indicated: (1) Clairvoyant smoother, (2) CUSUM, (3) STATESON, (4) WJ: Willsky-Jones, (5) PF (particle smoother), (6) conventional Kalman smoother and (7) IMM.

Remark 11 The state estimates approach that of the clairvoyant algorithm as the SNR increases. To understand the discrepancy in the plot, one must keep in mind the logarithmic scale, and that even for high SNR there will be occasional small jumps, that remain undetected by the algorithms that do not have access to the correct jump instances.

STATESON is in some sense the closest convex relaxation of (10). It is therefore not very surprising that it is doing so well. That STATESON is doing very well over 
the various $\sqrt{Q / R_{e}}$-values also motivates the suggested $\lambda$-choice in (28).

\subsection{Computational Complexity}

In the numerical illustrations, we used our own implementation of all algorithms except the IMM smoother, for which we used the implementation of [33]. A CVX implementation of STATESON was used, see also Section 7 . The computational times (in seconds) for one run in Example 10 are given in Table 1. None of the algorithms have been optimized for computational speed.

Table 1

Computation times (in seconds) for one run in Example 10. Clairvoyant CUSUM STATESON WJ PF Kalman IMM

$\begin{array}{lllllll}0.1 & 0.1 & 2.4 & 0.1 & 54 & 0.1 & 0.4\end{array}$

Remark 12 With unknown jump-times, (10) (with the correct $\lambda$-choice) or the filter banks would be unbeatable. Their combinatorial-complexity make them computationally infeasible though. In Example 10, $2^{99} \approx 10^{30}$ constrained least squares problems would have to be solved for each Monte-Carlo run.

\section{Extension to Nonlinear Models}

An extension to nonlinear systems is of interest since many systems are poorly described by linear approximations. We do this in an extended-Kalman-filter-like fashion and approximate the nonlinear system by a timevarying linear model. To get an initial state trajectory estimate, we use an Extended Kalman Filter (EKF). The algorithm is summarized in Algorithm 3.

Algorithm 3 State Estimation by Sum-of-Norms Regularization Using Nonlinear Models Given a nonlinear state space model and $\{(y(t), u(t))\}_{t=1}^{N}$. Let $\epsilon$ be a positive parameter. Then, for a chosen $\lambda$ :

(1) Find an initial state trajectory estimate by applying an extended Kalman filter.

(2) Create a time-varying approximation of the nonlinear system by linearizing around the computed state trajectory.

(3) Apply Algorithm 2 to obtain a new state estimate.

(4) Return to step (2) if necessary.

Example 13 A Nonlinear Example - A Pendulum

Consider the pendulum shown in Figure \%. Its dynamical behavior using a mass $m=1$ and a pole length $L=1$ is described by the nonlinear relation

$$
\frac{d}{d t}\left[\begin{array}{c}
\theta \\
d \theta / d t
\end{array}\right]=\left[\begin{array}{c}
d \theta / d t \\
-g \sin \theta
\end{array}\right]+\left[\begin{array}{c}
0 \\
F
\end{array}\right] .
$$

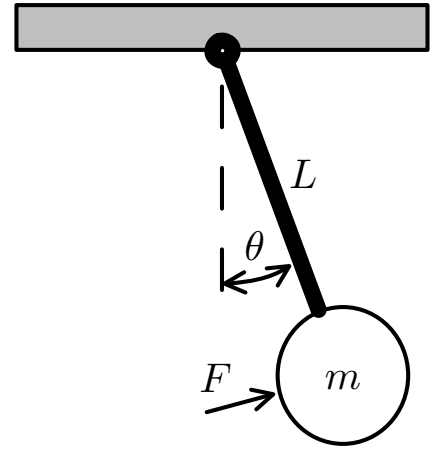

Fig. 7. Notation for the pendulum in Example 13.

$g$ is the gravitational constant $(g=9.81$ was used in the simulations). Using Euler integration with a time step of 0.005 , we obtain the time-discrete representation $\left(x_{1}=\theta, x_{2}=d \theta / d t\right)$

$\left[\begin{array}{l}x_{1}(t) \\ x_{2}(t)\end{array}\right]=\left[\begin{array}{c}x_{1}(t-1)+0.005 x_{2}(t-1) \\ x_{2}(t-1)-0.005 g \sin x_{1}(t-1)\end{array}\right]+\left[\begin{array}{c}0 \\ F(t)\end{array}\right]$.

Let us assume that we can measure the quantity

$$
y(t)=\sin x_{1}(t)+e(t), \quad e(t) \sim N(0,0.5)
$$

and that the system is driven by the process disturbance

$$
F(t)=w(t)+v(t), \quad w(t) \sim N(0,0.0005)
$$

and

$$
v(t)= \begin{cases}1 & \text { for } t=500 \\ 0 & \text { otherwise }\end{cases}
$$

$x_{1}(1)=\pi / 3$ and $x_{2}(1)=0$ were used to initialize the system and $y(t), t=1, \ldots, 1000$, observed, see the top plot of Figure 8. The estimate obtained by applying the EKF, step (1) of Algorithm 3, is given in the middle plot of Figure $8\left(\sin \hat{x}_{2}(t)\right.$ plotted). A linear time-varying representation of the pendulum was next computed around the x-estimate from the EKF (step (2) of Algorithm 3). Finally, using $\lambda$ as in (28), $\epsilon=10^{-4}$, and two iterations in Algorithm 3 (the criterion (24) was used, see Remark 5, due to the Gaussian component in the process disturbance), the estimate of $v$ given in the bottom plot of Figure 8 was obtained. As seen, the impulse at $t \approx 500$ was correctly detected.

\section{Conclusion}

A new formulation of the state estimation problem in the presence of abrupt changes has been presented. The proposed approach treats the state smoothing problem as a constrained least-squares problem with a sum-of-norms regularization, a convex formulation. A nice property of the proposed formulation is that it can be seen as an $\ell_{1}$ 

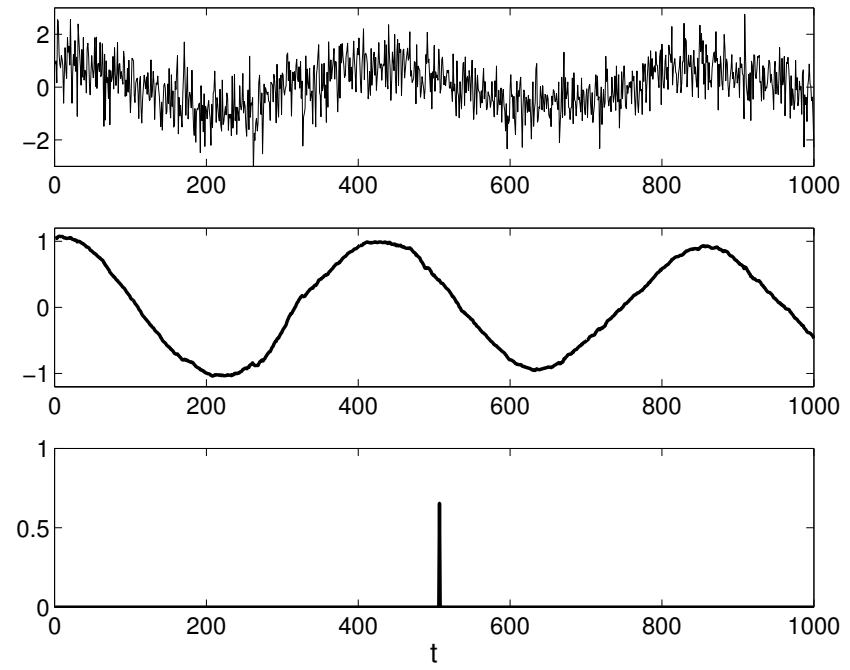

Fig. 8. Top plot shows the measured output $y(t)$. The middle plot shows $\sin x_{2}(t)$, with $x_{2}(t)$ obtained by applying an EKF to the data of the top plot (step (1) of Algorithm 3). Bottom plot gives the final estimate for $v$.

relaxation of the well known generalized likelihood ratio method by Willsky and Jones. Many other methods for state estimation in the presence of abrupt changes have been suggested in the literature. We have found in numerical examples that the suggested method shows very good performance in comparison with six other algorithms. Given that the proposed method is in some sense the closest convex relaxation of the optimal, but combinatorially forbidding, generalized likelihood ratio method by Willsky and Jones, this may not be that strange. Also, given that the method results in a convex optimization problem, the computational burden is reasonable. The extension to nonlinear models was also discussed and exemplified.

\section{A Proof of Proposition 6}

To verify our formula for $\lambda^{\max }$ we use convex analysis $[31,3,5]$. First note that

$$
\begin{aligned}
x(t) & =G v(t-1)+A x(t-1)+B u(t-1) \\
& =\sum_{r=1}^{t-1} A^{t-r-1}(G v(r)+B u(r))+A^{t-1} x(1) .
\end{aligned}
$$

Introduce

$$
\varepsilon_{t} \triangleq R_{e}^{-1 / 2}\left(y(t)-C\left(\sum_{r=1}^{t-1} A^{t-r-1} B u(r)+A^{t-1} x(1)\right)\right)
$$

and let $\bar{\varepsilon}_{t}$ be $\varepsilon_{t}$ evaluated at the $x(1)$ minimizing

$$
\min _{x(1)} \sum_{t=1}^{N}\left\|\varepsilon_{t}\right\|_{2}^{2}
$$

(19) can then be written as

$$
\begin{aligned}
\min _{\substack{x(1), \bar{v}(t), t \\
1, \ldots, N-1}} & \sum_{t=1}^{N}\left\|\bar{\varepsilon}_{t}-R_{e}^{-1 / 2} C \sum_{r=1}^{t-1} A^{t-r-1} G Q^{1 / 2} \bar{v}(r)\right\|_{2}^{2} \\
+\lambda & \sum_{t=1}^{N-1}\|\bar{v}(t)\|_{p}
\end{aligned}
$$

with $\bar{v}(t) \triangleq Q^{-1 / 2} v(t)$ and using (A.2). The subdifferential of $\|\bar{v}(t)\|_{p}$ evaluated at $\bar{v}(t)=0$ is the unit ball in the dual norm $\|\cdot\|_{q}, 1 / p+1 / q=1$. $\lambda^{\max }$ must therefore be give by

$$
\begin{aligned}
\lambda^{\max } & =\max _{k}\left\|\nabla_{\bar{v}(k)} \sum_{t=1}^{N}\right\| \bar{\varepsilon}_{t}-R_{e}^{-1 / 2} C \sum_{r=1}^{t-1} A^{t-r-1} G Q^{1 / 2} \bar{v}(r)\left\|\left._{2}^{2}\right|_{\bar{v} \equiv 0}\right\|_{q} \\
& =\max _{k}\left\|2 \sum_{t=k+1}^{N}\left(R_{e}^{-1 / 2} C A^{t-k-1} G Q^{1 / 2}\right)^{T} \bar{\varepsilon}_{t}\right\|_{q}
\end{aligned}
$$

\section{References}

[1] C. Andrieu, A. Doucet, S.S. Singh, and V.B. Tadic. Particle methods for change detection, system identification, and control. Proceedings of the IEEE, 92(3):423-438, March 2004.

[2] M. Basseville and I. V. Nikiforov. Detection of Abrupt Changes - Theory and Application. Prentice-Hall, Englewood Cliffs, NJ, 1993.

[3] D. P. Bertsekas, A. Nedic, and A. E. Ozdaglar. Convex Analysis and Optimization. Athena Scientific, 2003.

[4] H. A. P. Blom and Y. Bar-Shalom. The interacting multiple model algorithm for systems with Markovian switching coefficients. IEEE Transactions on Automatic Control, 33(8):780-783, August 1988.

[5] J. M. Borwein and A. S. Lewis. Convex Analysis and Nonlinear Optimization: Theory and Examples. CMS Books in Mathematics. Springer, 2000.

[6] S. Boyd, N. Parikh, E. Chu, B. Peleato, and J. Eckstein. Distributed optimization and statistical learning via the alternating direction method of multipliers. Technical report, Stanford University, January 2011.

[7] S. Boyd and L. Vandenberghe. Convex Optimization. Cambridge University Press, 2004.

[8] E. J. Candès, J. Romberg, and T. Tao. Robust uncertainty principles: Exact signal reconstruction from highly incomplete frequency information. IEEE Transactions on Information Theory, 52:489-509, February 2006.

[9] E. J. Candès, M. B. Wakin, and S. Boyd. Enhancing sparsity by reweighted $\ell_{1}$ minimization. Journal of Fourier Analysis and Applications, special issue on sparsity, 14(5):877-905, December 2008.

[10] D. L. Donoho. Compressed sensing. IEEE Transactions on Information Theory, 52(4):1289-1306, April 2006.

[11] D. Gabay and B. Mercier. A dual algorithm for the solution of nonlinear variational problems via finite element approximation. Computers \& Mathematics with Applications, 2(1):17-40, 1976. 
[12] R. Glowinski and A. Marrocco. Sur lapproximation par elements finis dordre un, et la resolution par penalisationdualite dune classe de problemes de Dirichlet nonlineaires. Rev. Francaise dAut., pages 41-76, 1975. Inf. Rech. Oper., $\mathrm{R}-2$.

[13] N.J. Gordon, D.J. Salmond, and A.F.M. Smith. Novel approach to nonlinear/non-Gaussian Bayesian state estimation. Radar and Signal Processing, IEE Proceedings F, 140(2):107-113, April 1993.

[14] M. Grant and S. Boyd. Graph implementations for nonsmooth convex programs. In V. D. Blondel, S. Boyd, and H. Kimura, editors, Recent Advances in Learning and Control, Lecture Notes in Control and Information Sciences, pages 95-110. Springer-Verlag Limited, 2008. http:// stanford.edu/ boyd/graph_dcp.html.

[15] M. Grant and S. Boyd. CVX: Matlab software for disciplined convex programming, version 1.21. http://cvxr.com/cvx, August 2010.

[16] F. Gustafsson. The marginalized likelihood ratio test for detecting abrupt changes. IEEE Transactions on Automatic Control, 41(1):66-78, 1996.

[17] F. Gustafsson. Adaptive Filtering and Change Detection. Wiley, New York, 2001.

[18] F. Gustafsson. Statistical Sensor Fusion. Studentlitteratur AB, 2010.

[19] T. Hastie, R Tibshirani, and J. Friedman. The Elements of Statistical Learning: Data Mining, Inference, and Prediction. Springer Series in Statistics. Springer New York Inc., New York, NY, USA, 2001.

[20] T. Kailath, A. H. Sayed, and B. Hassibi. Linear Estimation. Prentice-Hall, Englewood Cliffs, NJ, 2000.

[21] S.-J. Kim, K. Koh, S. Boyd, and D. Gorinevsky. $\ell_{1}$ trend filtering. SIAM Review, 51(2):339-360, 2009.

[22] S.-J. Kim, K. Koh, M. Lustig, S. Boyd, and D. Gorinevsky. An interior-point method for large-scale 11-regularized least squares. IEEE Journal of Selected Topics in Signal Processing, 1(4):606-617, December 2007.

[23] W. Koch. Fixed-interval retrodiction approach to Bayesian IMM-MHT for maneuvering multiple targets. IEEE Transactions on Aerospace and Electronic Systems, 36(1):214, January 2000.

[24] L. Ljung. System Identification - Theory for the User. Prentice-Hall, Upper Saddle River, N.J., 2nd edition, 1999.

[25] L. Ljung and T. Kailath. A unified approach to smoothing formulas. Automatica, 12(2):147-157, 1976.

[26] J. Löfberg. Yalmip : A toolbox for modeling and optimization in MATLAB. In Proceedings of the CACSD Conference, Taipei, Taiwan, 2004.

[27] H. Ohlsson, L. Ljung, and S. Boyd. Segmentation of ARX-models using sum-of-norms regularization. Automatica, 46(6):1107-1111, 2010.

[28] M. R. Osborne, B. Presnell, and B. A. Turlach. On the LASSO and its dual. Journal of Computational and Graphical Statistics, 9:319-337, 1999.

[29] E. S. Page. Continuous inspection schemes. Biometrika, 41(1/2):100-115, 1954.

[30] R. Patton, P. Frank, and R. Clark. Fault Diagnosis in Dynamic Systems - Theory and Application. Prentice Hall, 1989.

[31] R. T. Rockafellar. Convex Analysis. Princeton University Press, 1996.
[32] J. Roll. Piecewise linear solution paths with application to direct weight optimization. Automatica, 44:2745-2753, 2008.

[33] S. Särkkä and J. Hartikainen. EKF/UKF toolbox for Matlab 7.x, November 2007. Version 1.2.

[34] R. Tibsharani. Regression shrinkage and selection via the lasso. Journal of Royal Statistical Society B (Methodological), 58(1):267-288, 1996.

[35] A. Willsky. A survey of design methods for failure detection in dynamic systems. Automatica, 12:601-611, 1976.

[36] A. Willsky and H. Jones. A generalized likelihood ratio approach to the detection and estimation of jumps in linear systems. IEEE Transactions on Automatic Control, 21(1):108-112, February 1976.

[37] M. Yuan and Y. Lin. Model selection and estimation in regression with grouped variables. Journal of the Royal Statistical Society, Series B, 68:49-67, 2006.

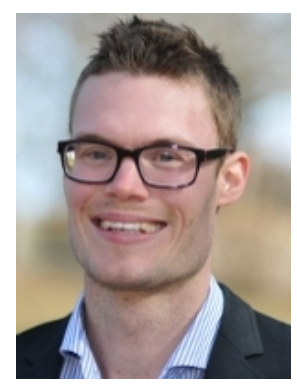

Henrik Ohlsson was born in Sweden in 1981. He received the M.Sc. degree in Applied Physics and Electrical Engineering in Oct. 2006 and his Ph.D. degree in Automatic Control in Nov. 2010, all from Linköping University, Sweden. He has held visiting positions at the University of Cambridge (UK) and the University of Massachusetts (USA). His research interests are mainly within the areas of system identification and machine learning. He is currently an Assistant Professor at Linköping University.

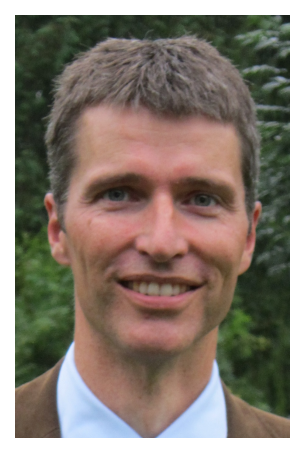

Fredrik Gustafsson is professor in Sensor Informatics at Department of Electrical Engineering, Linköping University, since 2005. He received the M.Sc. degree in Electrical Engineering 1988 and the Ph.D. degree in Automatic Control, 1992, both from Linköping University. During 1992-1999 he held various positions in automatic control, and 1999-2005 he had a professorship in Communication Systems. His research interests are in stochastic signal processing, adaptive filtering and change detection, with applications to communication, vehicular, airborne, and audio systems.

He has supervised $15 \mathrm{PhD}, 20$ licentiate and more than 170 master theses. He is the author of five books and over 170 conference papers, 60 journal papers and some twenty patents (h-index 30 in Google Scholar).

He is a co-founder of the companies NIRA Dynamics and Softube, developing signal processing software solutions for automotive and music industry, respectively. 
He was an associate editor for IEEE Transactions of Signal Processing 2000-2006 and is currently associate editor for IEEE Transaction of Aerospace and Electronic Systems and EURASIP Journal on Advances in Signal Processing. In 2004, he was awarded the Arnberg prize by the Royal Swedish Academy of Science (KVA) and in 2007 he was elected member of the Royal Academy of Engineering Sciences (IVA).

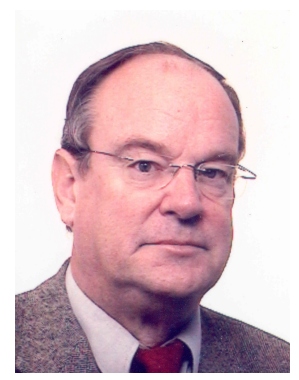

Lennart Ljung received his $\mathrm{PhD}$ in Automatic Control from Lund Institute of Technology in 1974. Since 1976 he is Professor of the chair of Automatic Control In Linkoping, Sweden, and is currently Director of the Stratetic Research Center "Modeling, Visualization and Information Integration" (MOVIII). He has held visiting positions at Stanford and MIT and has written several books on System Identification and Estimation. He is an IEEE Fellow, an IFAC Fellow and an IFAC Advisor as well as a member of the Royal Swedish Academy of Sciences (KVA), a member of the Royal Swedish Academy of Engineering Sciences (IVA), an Honorary Member of the Hungarian Academy of Engineering and a Foreign Associate of the US National Academy of Engineering (NAE). He has received honorary doctorates from the Baltic State Technical University in St Petersburg, from Uppsala University, Sweden, from the Technical University of Troyes, France, from the Catholic University of Leuven, Belgium and from Helsinki University of Technology, Finland. In 2002 he received the Quazza Medal from IFAC, in 2003 he recieved the Hendrik W. Bode Lecture Prize from the IEEE Control Systems Society, and he was the recepient of the IEEE Control Systems Award for 2007.

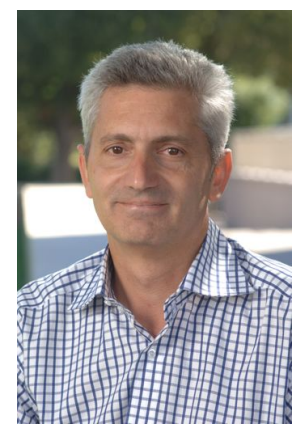

Stephen Boyd is the Samsung Professor of Engineering in the Electrical Engineering Department at Stanford University. His current interests include convex programming applications in control, signal processing, and circuit design. He received an $\mathrm{AB}$ degree in Mathematics, summa cum laude, from Harvard University in 1980, and a $\mathrm{PhD}$ in EECS from U. C. Berkeley in 1985. He is the author of Linear Controller Design: Limits of Performance (with Craig Barratt, 1991), Linear Matrix Inequalities in System and Control Theory (with L. El Ghaoui, E. Feron, and V. Balakrishnan, 1994), and Convex Optimization (with Lieven Vandenberghe, 2004). 